\title{
Assessment of relaxin levels in pregnant women with gestational diabetes
}

I Zaman , R Swaminathan, A Brackenridge , A Sankaralingam , BM McGowan

Department of Endocrinology, Guy's and St Thomas' NHS Foundation Trust, London, SE1 9RT, United Kingdom

\section{Introduction:}

\section{- Gestational diabetes mellitus (GDM) is an} important co-morbidity of pregnancy, with insulin resistance a key contributor in its underlying pathology.

Relaxin belongs to a family of structurally related peptides called the Insulin super family and has a structure similar to insulin (Figure 1).

- Relaxin is a hormone secreted mainly in pregnancy with plasma levels highest in the first trimester, reaching up to $900 \mathrm{pg} / \mathrm{ml}$ by 10 weeks and decreasing thereafter, with lowest levels in the third trimester, at approximately $500 \mathrm{pg} / \mathrm{ml}$ after 24 weeks. Relaxin has several effects on reproductive and non-reproductive system, and it is essential for maternal adaptations to pregnancy.

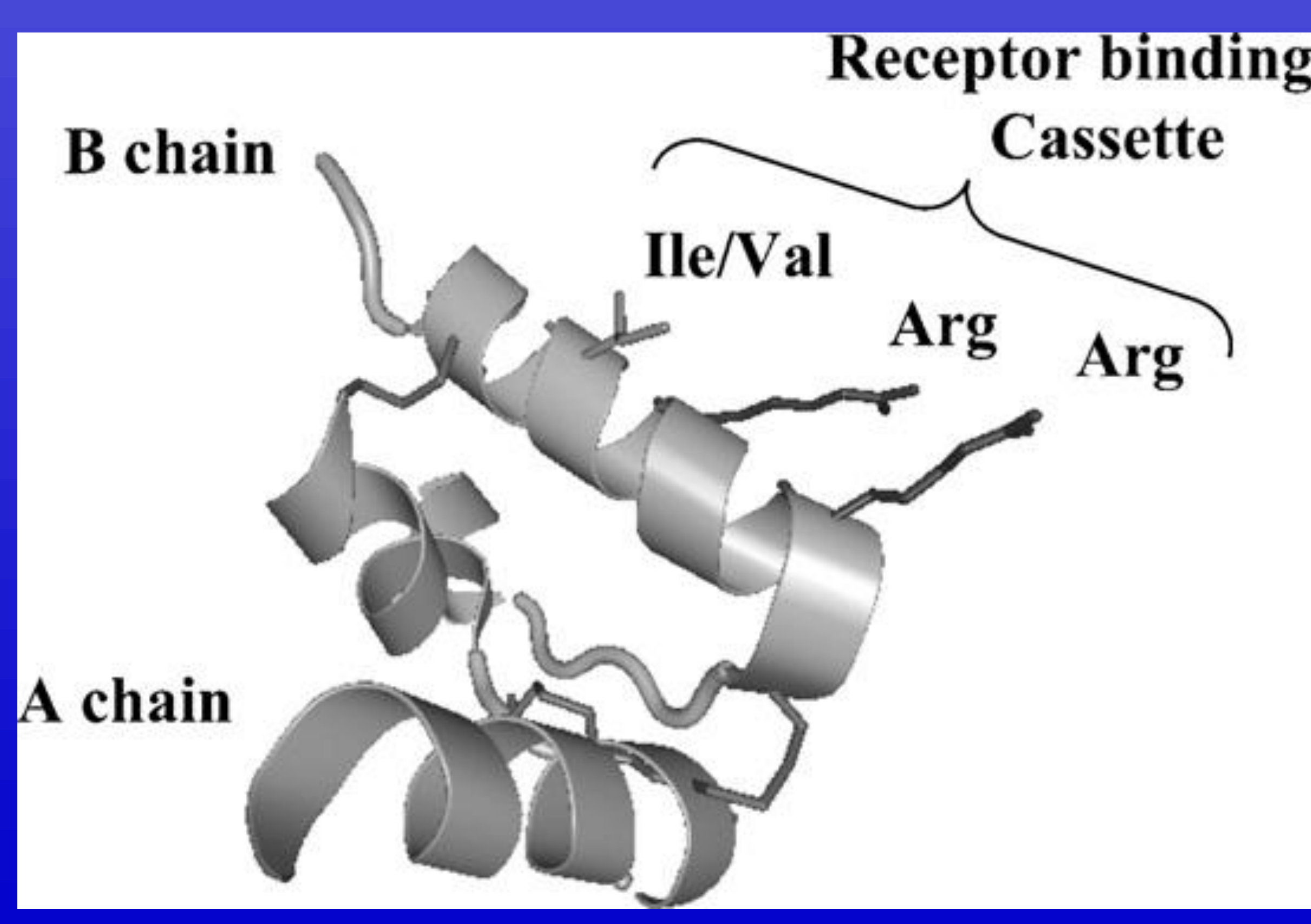

Figure 1. Relaxin structure: $A$ chain and $B$ chain interconnected by disulphide bridges. Receptor binding cassette enables relaxin to bind to its receptor

- Previous studies have suggested that levels of plasma relaxin may be positively correlated with insulin sensitivity ${ }^{1,2}$.

- This led us to hypothesize that relaxin may play a role in the pathogenesis of insulin resistance in women with gestational diabetes.

\section{Aim:}

- To assess relaxin levels in pregnant women with gestational diabetes (GDM) compared to a control group of healthy pregnant women in the third trimester of pregnancy.

- To determine the correlation between relaxin and insulin sensitivity \& $\beta$-cell function

\section{Design and Methods:}

Twenty-six GDM subjects and 27 healthy controls were recruited, matched for gestation term and age. A blood sample was obtained to determine plasma relaxin levels during the $3^{\text {rd }}$ trimester of pregnancy.

Plasma relaxin was measured by ELISA.

For 15 subjects who underwent a fasting oral glucose tolerance to test for GDM, insulin sensitivity and $\beta$-cell function was also assessed. This was calculated using a HOMA (Homeostatic model assessment) calculator which used fasting glucose, fasting insulin and C-peptide concentrations.

- A Mann- Whitney U test was used for statistical analysis, including testing for differences in baseline characteristics between the two groups (Age, HbA1C, pregnancy duration). Correlation between relaxin and other variables was determined using The Spearman's Rank correlation coefficient.

\section{Results}

- Plasma relaxin levels in the GDM group were higher compared to the healthy control group (median relaxin concentration $667.5 \mathrm{pg} / \mathrm{ml}$ in GDM group vs $439 \mathrm{pg} / \mathrm{ml}$ in control group), but this did not reach statistical significance $(p=0.15)$ (Figure 2)

- Relaxin concentration was positively correlated with insulin sensitivity and inversely correlated with $\beta$-cell function, but this did not reach statistical significance (Table 1).

- There was no significant difference in age and pregnancy duration between the two groups. No significant correlation was found between relaxin concentration and other variables - Age, pregnancy duration (gestation week), HbA1C.

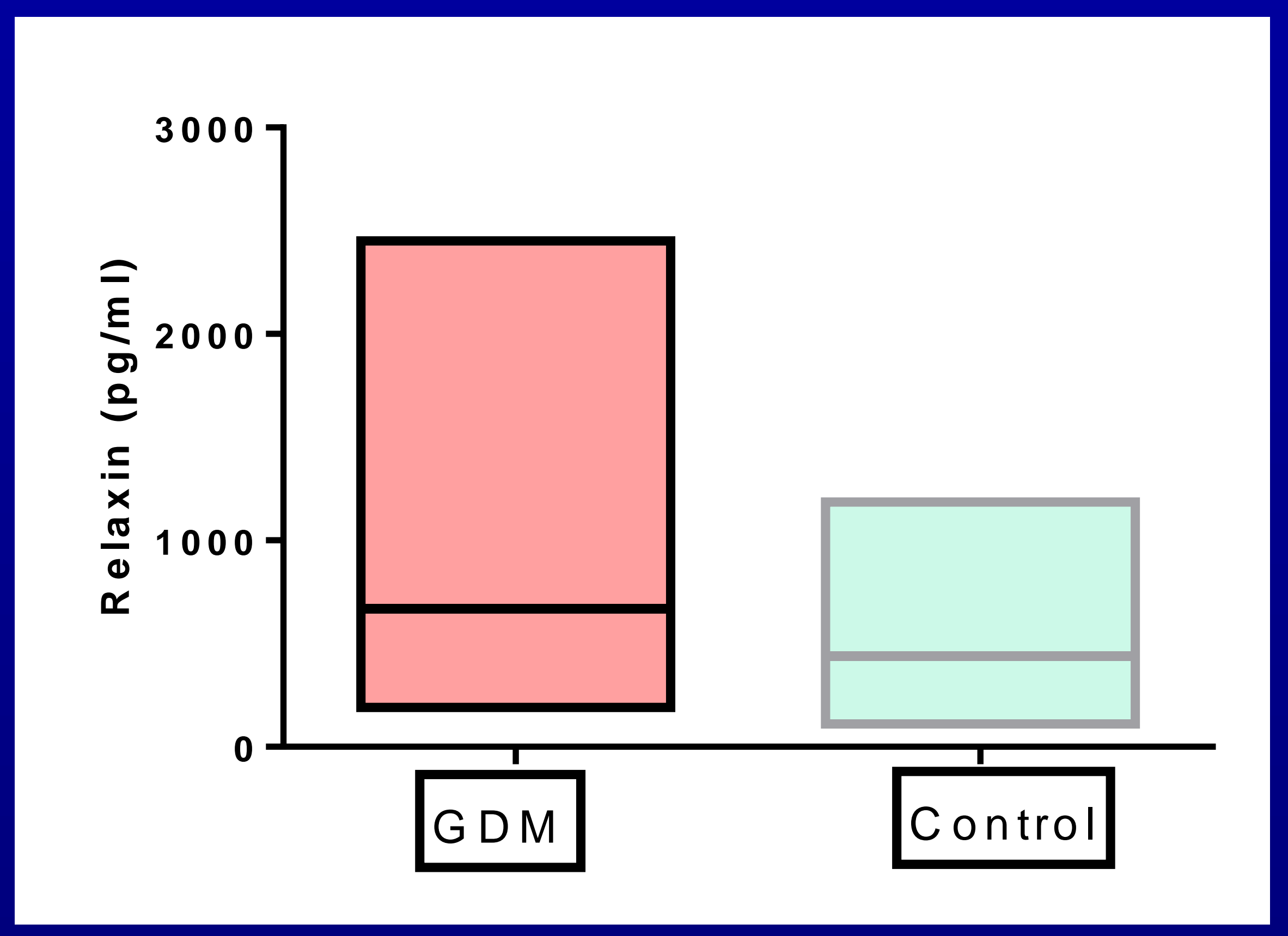

Figure 2: Relaxin concentrations between GDM $(n=26) \quad V s$ Control $(n=27)$. Median relaxin concentration is denoted by the horizontal line within the bar chart, $p=0.15$.

\begin{tabular}{|c|c|c|}
\hline & $R$ value & $P$ value \\
\hline IS $^{1}$ & 0.39 & 0.11 \\
\hline $\begin{array}{l}\beta \text {-cell } \\
\text { function } 1\end{array}$ & -0.39 & 0.10 \\
\hline IS $^{2}$ & 0.25 & 0.31 \\
\hline $\begin{array}{l}\beta \text {-cell } \\
\text { function }{ }^{2}\end{array}$ & -0.35 & 0.15 \\
\hline
\end{tabular}

Table 1: Correlation between relaxin and insulin sensitivity \& $\beta$-cell function. IS 1 and $\beta$-cell function ${ }^{1}=$ Calculated from fasting glucose + fasting insulin values. $I^{2}$ and $\beta$-cell function ${ }^{2}=$ calculated from fasting glucose + fasting C-peptide values. $\mathrm{R}=$ Correlation, $\mathrm{P}=$ Significance of correlation. $\mathrm{P}<0.05$ is defined as clinically significant

- Plasma relaxin levels were higher in women with GDM compared to healthy pregnant women, although this did not reach statistical significance.

- Increased relaxin levels in GDM may be a result of the body's compensatory mechanism to overcome increased insulin resistance.

- More studies are required to understand whether relaxin plays a role in the pathogenesis of gestational diabetes and insulin resistance.

\section{References:}

1) Olefsky JM, Saekow M, Kroc RL. Potentiation of insulin binding and insulin action by purified porcine relaxin. Ann N Y Acad Sci 1982; 80: 200-16

2) Barbara Szepietowska , Gorska M, Szelachowska M. (2008). Plasma relaxin concentration is related to betacell function and insulin sensitivity in women with type 2 diabetes mellitus. Diabetes research and clinical practice;79: e 1 - e 3

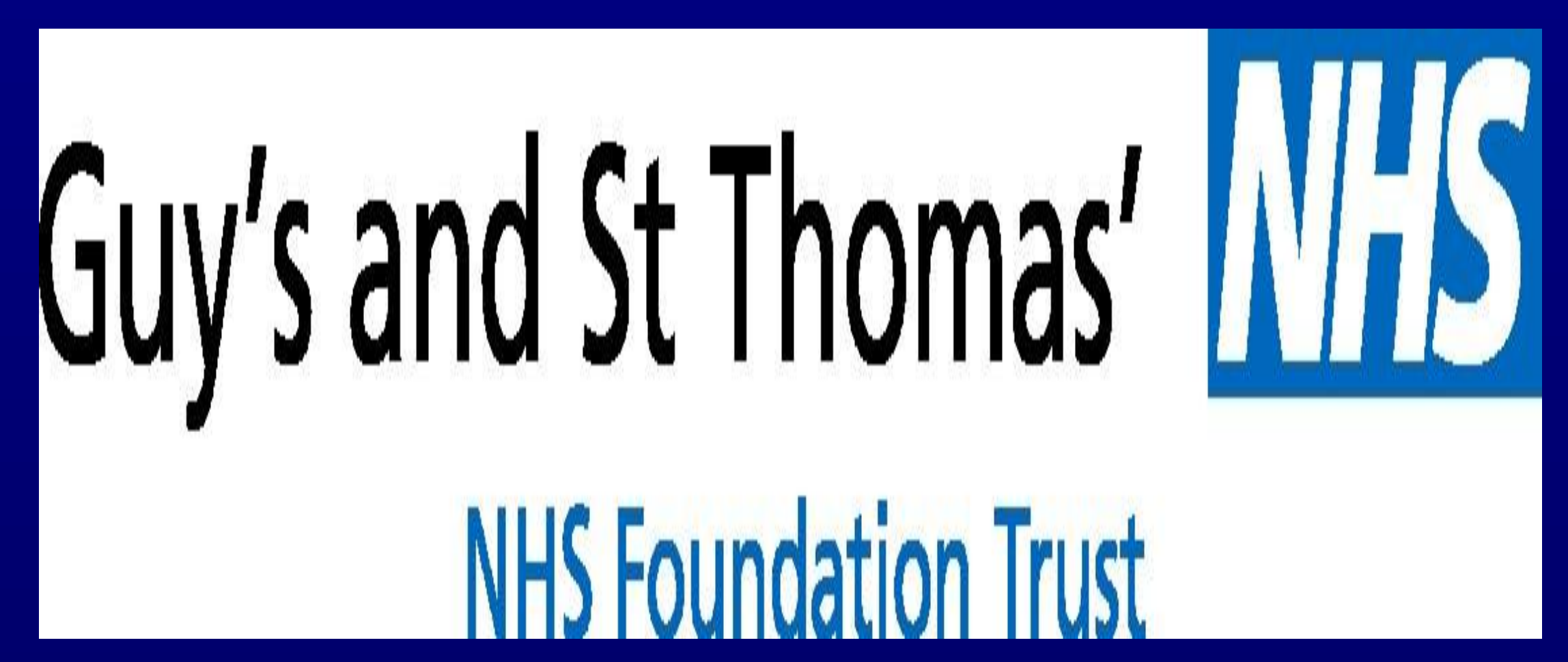

